\title{
Electromagnetics
}

\section{Circuit Model for Power Link Budget Between Two Horizontal Loops Placed on Seabed}

\author{
Eugenio Jiménez-Yguácel* \\ Instituto Desarrollo Tecnológico e Innovación en Comunicaciones, Universidad de Las Palmas de Gran Canaria, 35001 Las Palmas de \\ Gran Canaria, Spain \\ * Member, IEEE
}

Received 13 Jan 2020, revised 9 Mar 2020, accepted 16 Mar 2020, published 23 Mar 2020, current version 20 Apr 2020.

Abstract-In this letter, we examine underwater wireless communications using electromagnetic waves in marine shallow waters. Due to the particular environment under study (low frequencies, short distances, and small loop antennas), a magnetoquasi-static approach is used instead of full-wave analysis. The electric field generated by a constant current circular loop placed on the interface between two conductors is analytically calculated. Using this electric field, the mutual coupling coefficient between the two loops is calculated, and a simple circuit model for transmitting energy from one loop to another is presented using a transformerlike element. Simulations using the circuit model and a commercial full-wave simulator are carried out, and their results are compared. Very good agreement is observed between both results.

Index Terms—Electromagnetics, underwater communications, magnetic field communications, sea water seabed interface, loop antenna, circuit model.

\section{INTRODUCTION}

Underwater communications have been theoretically studied since early radio days. Basic theory is based upon the resolution of the socalled half-space Sommerfeld problem. The exact, full-wave, analysis of this problem led to the publication of a great number of articles during the first half of the 20th century. These findings have been summarized in the classical book [Bagnos 1966]. Lately, the extension of the two-layered case to three-layered and multilayered cases led to the publication of a long series of papers. The details of the research findings were well summarized in two books [King 1992, Wait 1996]. Full-wave analysis is valid for all frequencies and distances, but its mathematical complexity makes it difficult for practical applications. From an engineering point of view, something simple, such as a circuit model using lumped elements, would be desirable.

Electromagnetic underwater communication at short distances in shallow seawaters is a very interesting subject because it can be an alternative to optical or acoustic communications. In shallow seawater environments, the water is often turbid, and this makes it difficult to use light for wireless communications. Acoustic communications are widely used for long distances in deep waters, but they have some drawbacks, especially in shallow seawaters, due to time-varying multipath propagation and limited bandwidth ( 5 to $7 \mathrm{kHz}$ ).

Electromagnetic waves are greatly attenuated as they travel through seawater, due to the water conductivity. This attenuation increases with frequency, so very low frequencies have to be used to get a reliable communication ( 1 to $50 \mathrm{kHz}$ ). If short-range communications are going to be carried out using low frequencies and electrically small loop antennas, we can use the magnetoquasi-static (MQS) approach (for short) instead of full-wave analysis [Parise 2015]. The main advantage

Corresponding author: Eugenio Jiménez-Yguácel (e-mail: eugenio.jimenez@ ulpgc.es).

Digital Object Identifier 10.1109/LMAG.2020.2982610

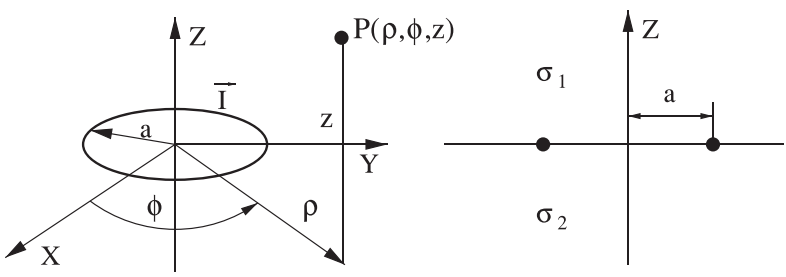

Fig. 1. Geometry of the problem.

of using the MQS approach is that we can extract an equivalent circuit for the radio link, which simplifies the study of the link budget.

\section{PROBLEM FORMULATION}

Consider a small current loop lying on the interface between two conducting medium, as shown in Fig. 1. The electric conductivity in each medium is denoted as $\sigma_{1}$ and $\sigma_{2}$, and the magnetic permeability is assumed to be $\mu_{0}$. The current flowing through the wire can be expressed as [Hurley 1995, Acero 2006]

$$
\mathbf{I}=I_{\phi} \delta(\rho-a) \delta(z) \hat{\phi} .
$$

Maxwell equations for a conductor without sources and using MQS are

$$
\nabla \times \mathbf{H}=\mathbf{J} \quad \nabla \times \mathbf{E}=-j \omega \mu \mathbf{H} \quad \mathbf{J}=\sigma \mathbf{E} .
$$

Due to the geometry of the source, the problem has cylindrical symmetry around the $Z$-axis and the electric field has only the $\hat{\phi}$ component. Under those assumptions, the electric-field equation is written as

$$
\frac{\partial^{2} E_{\phi}}{\partial z^{2}}+\frac{1}{\rho} \frac{\partial}{\partial \rho}\left(\rho \frac{\partial E_{\phi}}{\partial \rho}\right)-\frac{E_{\phi}}{\rho^{2}}=j \omega \mu \sigma E_{\phi} .
$$

Bessel operator $\Delta_{v}$ is defined as

$$
\Delta_{v}=\frac{1}{\rho} \frac{\partial}{\partial \rho}\left(\rho \frac{\partial}{\partial \rho}\right)-\left(\frac{v}{\rho}\right)^{2}
$$


and then (3) is written as

$$
\frac{\partial^{2} E_{\phi}}{\partial z^{2}}+\Delta_{1} E_{\phi}=j \omega \mu \sigma E_{\phi} .
$$

When dealing with problems with cylindrical symmetry, it is very useful to use Hankel transform [Piessens 2010]. This transform and its inverse are defined as

$$
\begin{aligned}
& \tilde{f}(\alpha) \equiv \mathcal{H}_{v}[f(\rho)] \equiv \int_{0}^{\infty} \rho f(\rho) J_{v}(\alpha \rho) d \rho \\
& f(\rho) \equiv \mathcal{H}_{v}^{-1}[\tilde{f}(\alpha)] \equiv \int_{0}^{\infty} \alpha \tilde{f}(\alpha) J_{v}(\alpha \rho) d \alpha .
\end{aligned}
$$

The Hankel transform of the Bessel operator is easily calculated as

$$
\mathcal{H}_{v}\left[\Delta_{v} f(\rho)\right]=-\alpha^{2} \tilde{f}(\alpha) .
$$

Equation (3) is finally written in the transformed domain as

$$
\frac{\partial^{2} \tilde{E}_{\phi}}{\partial z^{2}}=\left(\alpha^{2}+j \omega \mu \sigma\right) \tilde{E}_{\phi}
$$

Defining $k_{1}=\sqrt{\alpha^{2}+j \omega \mu \sigma_{1}}, k_{2}=\sqrt{\alpha^{2}+j \omega \mu \sigma_{2}}$, and choosing the branch cut of the square root so $\Re e\left(k_{i}\right)>0$, the solutions to (9) in each of the half-spaces are

$$
\tilde{E}_{\phi}= \begin{cases}A e^{-k_{1} z} & z>0 \\ B e^{k_{2} z} & z<0 .\end{cases}
$$

Boundary conditions for $\tilde{\mathbf{E}}$ in $z=0$ lead to $A=B$, and boundary conditions for $\tilde{\mathbf{H}}$ in $z=0$ link its radial components with the current lying on the interface. The Hankel transform of the current is

$$
\tilde{\mathbf{I}}(\alpha)=\mathcal{H}_{1}[\mathbf{I}(\rho)]=I_{\phi} a J_{1}(\alpha a) \delta(z) \hat{\phi}
$$

We obtain the boundary condition for $\tilde{\mathbf{H}}$ integrating along the $z$-axis from a point infinitesimally close to $z=0$ in the lower half-space to another point infinitesimally close to $z=0$ in the upper half-space

$$
\tilde{H}_{\rho_{+}}-\tilde{H}_{\rho_{-}}=I_{\phi} a J_{1}(\alpha a) .
$$

The radial component of $\tilde{\mathbf{H}}$ is calculated from $\tilde{E_{\phi}}$

$$
\tilde{H}_{\rho}=\frac{1}{j \omega \mu} \frac{\partial \tilde{E}_{\phi}}{\partial z} .
$$

Finally, the electric field in the transformed domain is

$$
\tilde{\mathbf{E}}= \begin{cases}-\frac{j \omega \mu}{k_{1}+k_{2}} I_{\phi} a J_{1}(\alpha a) e^{-k_{1} z} \hat{\phi} & z>0 \\ -\frac{j \omega \mu}{k_{1}+k_{2}} I_{\phi} a J_{1}(\alpha a) e^{k_{2} z} \hat{\phi} & z<0 .\end{cases}
$$

The electric field $\mathbf{E}$ is obtained by taking the inverse transform in (14)

$$
\mathbf{E}= \begin{cases}-j \omega \mu I_{\phi} a \hat{\phi} \int_{0}^{\infty} \frac{e^{-k_{1} z}}{k_{1}+k_{2}} J_{1}(\alpha a) J_{1}(\alpha \rho) \alpha d \alpha & z>0 \\ -j \omega \mu I_{\phi} a \hat{\phi} \int_{0}^{\infty} \frac{e^{k_{2} z}}{k_{1}+k_{2}} J_{1}(\alpha a) J_{1}(\alpha \rho) \alpha d \alpha & z<0 .\end{cases}
$$

\section{MUTUAL INDUCTANCE OF TWO COPLANAR CIRCULAR LOOPS}

The geometric parameters of two coplanar circular loops are shown in Fig. 2. Both loops are lying on the $X Y$ plane between two conducting media of conductivities $\sigma_{1}$ and $\sigma_{2}$ as in Fig. 1 .

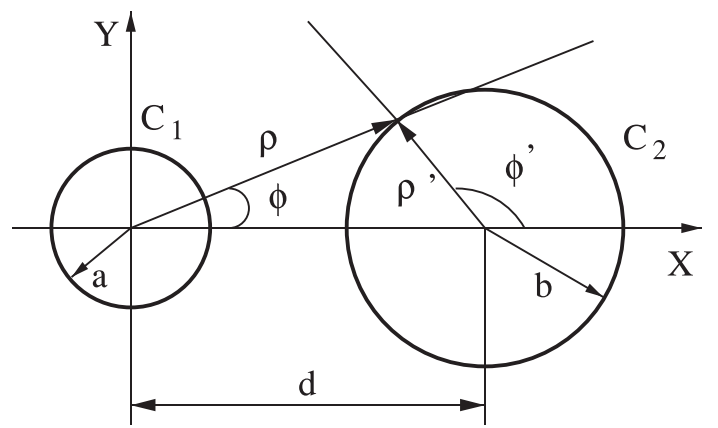

Fig. 2. Two loops placed on the interface between two media.

The electromotive force (EMF) $\epsilon$ generated in loop $C_{2}$ due to the current flowing through loop $C_{1}$ is obtained using the field $\mathbf{E}$ generated by $C_{1}$

$$
\epsilon=-\oint_{C_{2}} \mathbf{E} \cdot \mathbf{d l}
$$

Using polar coordinates $\mathbf{d} \mathbf{l}=\rho^{\prime} \hat{\phi}^{\prime} d \phi^{\prime}$ and taking into account that in our problem $\mathbf{E}$ field has only $\hat{\phi}$ component, (16) gives

$$
\epsilon=-\int_{0}^{2 \pi} b E_{\phi}\left(\rho^{\prime}=b\right) \hat{\phi}^{\prime} \cdot \hat{\phi} d \phi^{\prime} \text { being } \hat{\phi}^{\prime} \cdot \hat{\phi}=\cos \left(\phi-\phi^{\prime}\right) .
$$

The mutual inductance $M$ is calculated as

$$
\frac{\epsilon}{I_{\phi}}=j \omega M
$$

When the noncoaxial loops are in free space, the method to calculate $M$ is shown in Conway [2007]. In our case, the method is slightly different because the loops are placed on the interface of two conducting media. But, as in Conway [2007], $M$ only depends on the electric field in $z=0$. After some work, $M$ can be written in the form

$$
M=2 \mu a b \pi \int_{0}^{\infty} \frac{\alpha J_{0}(\alpha d) J_{1}(\alpha a) J_{1}(\alpha b)}{\sqrt{\alpha^{2}+j \omega \mu \sigma_{1}}+\sqrt{\alpha^{2}+j \omega \mu \sigma_{2}}} d \alpha .
$$

This is a new unpublished result.

The $M$ coefficient calculated using (19) is a complex number with real and imaginary parts. This is due to the finite conductivities $\sigma_{1}$ and $\sigma_{2}$. In the following sections, we are going to study real and imaginary parts of (19).

\section{A. Real Part of $M$}

We are not going to take into account the Bessel functions to define the real part of the integrand of (19) as

$$
f(\alpha)=\Re e\left[\frac{\alpha}{\sqrt{\alpha^{2}+j \omega \mu \sigma_{1}}+\sqrt{\alpha^{2}+j \omega \mu \sigma_{2}}}\right] .
$$

Defining $\alpha_{\delta}=\delta \sqrt{\omega \mu \sigma_{\delta}}$ with $\sigma_{\delta}=\max \left(\sigma_{1}, \sigma_{2}\right)$ and forcing $\sigma_{1}=\sigma_{2}=$ $\sigma_{\delta}(20)$ gives

$$
f\left(\alpha_{\delta}\right)=\frac{1}{2 \sqrt{2}} \delta \sqrt{\frac{\delta^{2}+\sqrt{1+\delta^{4}}}{1+\delta^{4}}} .
$$

A plot of (21) is shown in Fig. 3

Given $\omega, \mu, \sigma_{1}$, and $\sigma_{2}$, we can always choose a value for $\delta$ so that $f(\delta)$ will be as close to $1 / 2$ as needed. Then, the real part of (19) can 


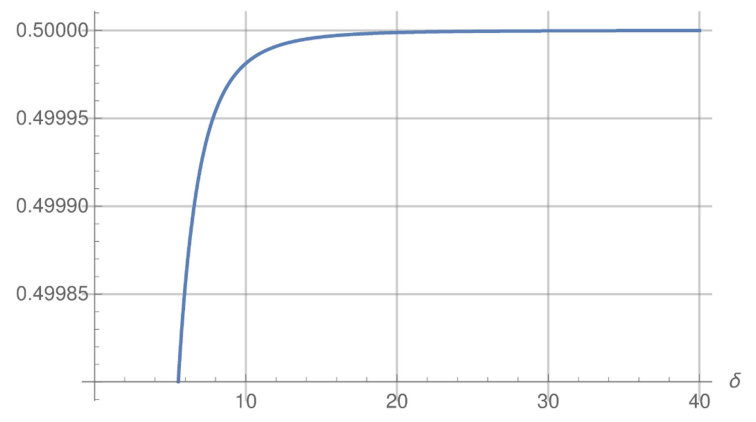

Fig. 3. Plot of $f\left(\alpha_{\delta}\right)$ versus $\delta$.

be approximated as

$$
\begin{aligned}
& \int_{0}^{\infty} f(\alpha) J_{0}(\alpha d) J_{1}(\alpha a) J_{1}(\alpha b) d \alpha \\
& \quad \approx \int_{0}^{\alpha_{\delta}}\left[f(\alpha)-\frac{1}{2}\right] J_{0}(\alpha d) J_{1}(\alpha a) J_{1}(\alpha b) d \alpha \\
& \quad+\frac{1}{2} \int_{0}^{\infty} J_{0}(\alpha d) J_{1}(\alpha a) J_{1}(\alpha b) d \alpha .
\end{aligned}
$$

Here, $\alpha_{\delta}$ has to be chosen depending on $\omega, \mu, \sigma_{1}, \sigma_{2}$, and $\delta$. Numerical experiments show that $\delta=25$ is enough.

The integral in the finite domain can be numerically evaluated using a numerical quadrature, such as QAG from the numerical quadrature library QUADPACK [Piessens 1983] or using a tool, such as Mathematica. ${ }^{1}$

The integral in the semi-infinite domain is the same that appears when calculating the coupling coefficient in the free space [Conway 2007]. In the limiting case, when $\sigma_{1}=\sigma_{2}=0$, the first integral vanishes and it only remains the free-space coupling coefficient.

\section{B. Imaginary Part of $M$}

The imaginary part of $M$, without Bessel functions, is defined as

$$
g(\alpha)=\Im m\left[\frac{\alpha}{\sqrt{\alpha^{2}+j \omega \mu \sigma_{1}}+\sqrt{\alpha^{2}+j \omega \mu \sigma_{2}}}\right] .
$$

Using the same parameter $\alpha_{\delta}$, the function that represents the imaginary part of the integrand is

$$
g\left(\alpha_{\delta}\right)=-\frac{1}{2 \sqrt{2}} \frac{\delta}{\left(1+\delta^{4}\right)\left(\delta^{2}+\sqrt{1+\delta^{4}}\right)} .
$$

A plot of (24) is shown in Fig. 4

As it happened with the real part of $M$, we can always choose a value for $\delta$ so $g(\delta)$ will be as close to 0 as needed. Then, the imaginary part of (19) can be approximated as

$$
\begin{aligned}
& \int_{0}^{\infty} g(\alpha) J_{0}(\alpha d) J_{1}(\alpha a) J_{1}(\alpha b) d \alpha \\
& \quad \approx \int_{0}^{\alpha_{\delta}} g(\alpha) J_{0}(\alpha d) J_{1}(\alpha a) J_{1}(\alpha b) d \alpha
\end{aligned}
$$

In the limiting case, when $\sigma_{1}=\sigma_{2}=0$, the integral vanishes and $M$ has no imaginary part.

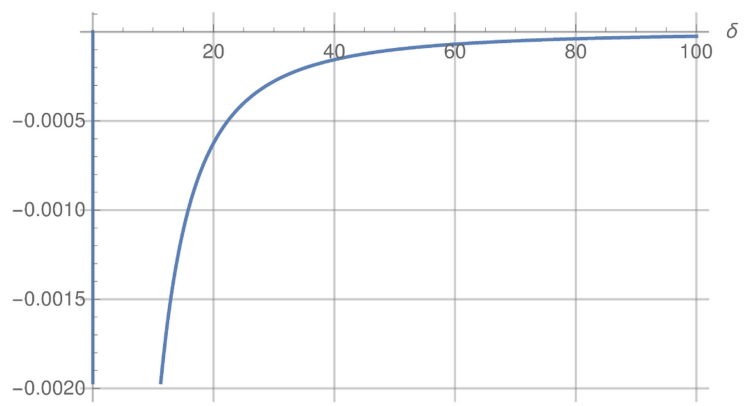

Fig. 4. Plot of $g\left(\alpha_{\delta}\right)$ versus $\delta$.

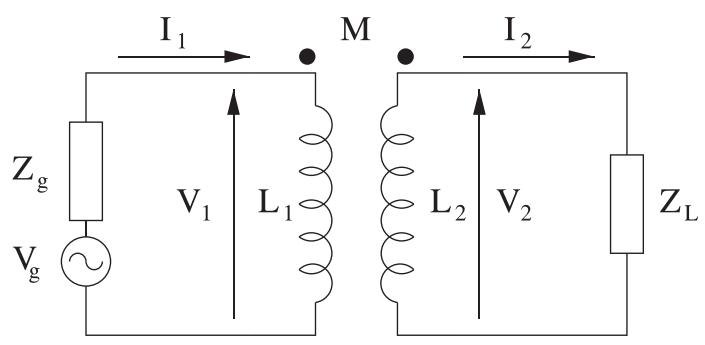

Fig. 5. Circuit model.

\section{CIRCUIT MODEL}

The equivalent circuit for two coupled loops [Domingo 2012] is shown in Fig. 5. $L_{i}$ is the self-inductance of the loops, and $M$ is the mutual inductance between them. Loops are assumed to be lossless.

The equations that relate currents and voltages are

$$
\begin{aligned}
& V_{1}=j \omega L_{1} I_{1}-j \omega M I_{2} \\
& V_{2}=j \omega M I_{1}-j \omega L_{2} I_{2} .
\end{aligned}
$$

The attenuation is defined as the ratio between the power dissipated into the load $P_{L}$ and the available power of the generator $P_{g}$

$$
\alpha=\frac{P_{L}}{P_{g}} \quad P_{L}=\frac{1}{2} \operatorname{Re}\left[V_{2} I_{2}^{*}\right] \quad P_{g}=\frac{V g^{2}}{8 \operatorname{Re}\left[Z_{g}\right]} .
$$

This definition is just the parameter $\left|s_{21}\right|^{2}$ of the transformer if the $S$-parameters are defined using $Z_{g}$ and $Z_{L}$ as reference impedances. To simplify, we assume that $Z_{g}=Z_{L}=Z_{0}$ as in most radio-frequency applications, and we assume that both loops are identical $\left(L_{1}=L_{2}=\right.$ $L$ ). The coupling coefficient $M$ has real and imaginary parts so $M=$ $M_{r}+j M_{i}$.

The expression that gives the attenuation is a bit long. It can be simplified if we assume that load currents have no influence in voltage $V_{1}$ so $V_{1}=j \omega L I_{1}$. In this case, the formula for the attenuation is

$$
\frac{P_{L}}{P_{d g}}=\frac{4 Z_{0}^{2} \omega^{2}\left(M_{r}^{2}+M_{i}^{2}\right)}{\left(\omega^{2} L^{2}+Z_{0}^{2}\right)^{2}} .
$$

\section{SIMULATIONS}

In this section, we are going to calculate the attenuation using (29), and we are going to compare the results with other results from a fullwave simulator. The numerical simulations will be performed using FEKO. $^{2}$ 


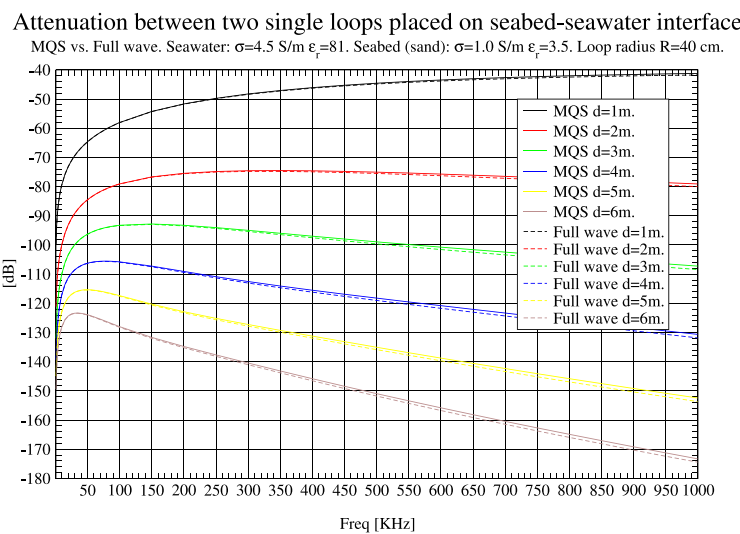

Fig. 6. Attenuation between single loops. MQS versus full wave.

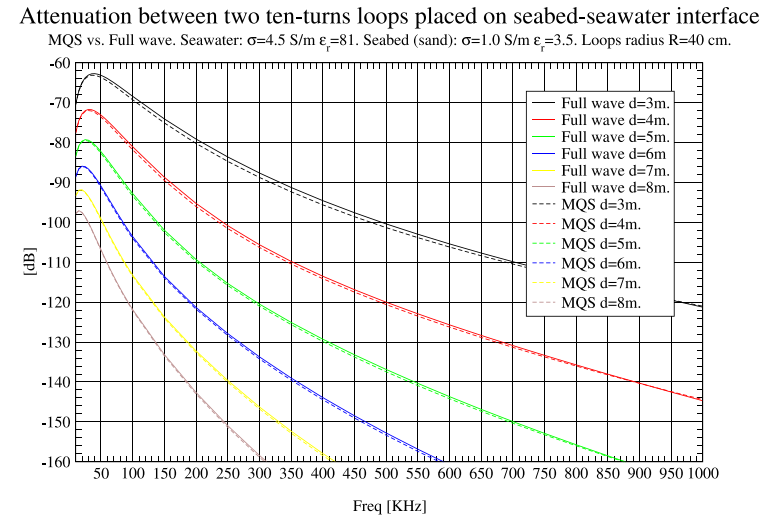

Fig. 7. Attenuation between coils. MQS versus full wave.

\section{A. Single-Loop Simulations}

The loops have a radius of $R=40 \mathrm{~cm}$, and they are made of isolated wire with radius $r=1.5 \mathrm{~mm}$. The loops have an inductance of $L=2.5 \mu \mathrm{H}$. Load and generator impedances are $Z_{0}=50 \Omega$. Both loops are placed laying on the interface between seawater $\left(\epsilon_{r}=\right.$ $\left.81, \sigma_{1}=4.5 \mathrm{~S} / \mathrm{m}\right)$ and seabed $\left(\epsilon_{r}=3.5, \sigma_{2}=1.0 \mathrm{~S} / \mathrm{m}\right)$. The electrical parameters of seawater and seabed are taken from Cella [2009].

The results obtained using both methods are shown in Fig. 6.

\section{B. Extension to Coils With $N$ Turns}

In practice, loop antennas use to have more than one turn in order to increase the signal level. The number of turns modifies two parameters in (29): the self-inductance $L$ and the mutual inductance $M$.

Using a simplified model, we can assume that the current flowing through $C_{1}$ (see Fig. 2) is $N_{1} I \phi$ with $N_{1}$ being the number of turns of coil $C_{1}$. We can assume, too, that the EMF induced in $C_{2}$, a coil with $N_{2}$ turns, is $N_{2}$ times the EMF for the single loop. Under these assumptions, $M_{\text {coil }}=N_{1} N_{2} M_{\text {loop }}$.

The calculation of the self-inductance of the coil $L_{\text {coil }}$ is more complicated because it depends on the way the turns are placed: single layer or multilayer. To keep the model simple, we assume that for the frequencies of interest, the inductance of the coil can be approximated by the inductance of the coil in free space. In our simulations, the formula for the inductance of the coil we are going to use is taken from Weaver [2020]

$$
\begin{aligned}
& L_{\text {coil }}=\mu N^{2} R \\
& \quad \times\left(\log \left(\frac{\pi R}{l}+1\right)+\frac{1}{\frac{0.4409 l^{2}}{R^{2}}+\frac{1.7185 l}{R}-\frac{0.47}{\left(\frac{2 R}{l}+0.755\right)^{1.44}}+2.3004}\right)
\end{aligned}
$$

where $l$ is the length of the coil, $R$ is the radius of the coil, and $N$ is the number of turns. In our simulations, $N=10, r=1.5 \mathrm{~mm}, l=5 \mathrm{~cm}$, and $R=40 \mathrm{~cm}$.

Full-wave simulations using FEKO and MQS approximation had been carried out, and the results are shown in Fig. 7

\section{CONCLUSION}

A simple circuit model for the electromagnetic coupling between two horizontal loops immersed in seawater and placed on the seabed has been presented in this letter. The circuit has been analytically obtained using MQS approximation. The attenuation between a generator and a load using the loops as antennas has been calculated using the circuit model. The results has been compared with those obtained using a full-wave simulator. A very good agreement has been observed between the results from the circuit model and the results from the full-wave simulator. This circuit model can be used to evaluate path losses in electromagnetic underwater communications and to predict optimal working frequencies for a given distance between antennas.

\section{ACKNOWLEDGMENT}

The author thanks Prof. J. M. Rebollar for the useful discussions and valuable comments. This work was supported by the Ministerio de Economía y Competitividad, Madrid, Spain, under Public Contract TEC2016-76038-C3.

\section{REFERENCES}

Acero J, Alonso R, Barragan L A, Burdio J M (2006), "Modeling of planar spiral inductors between two multilayer media for induction heating applications," IEEE Trans. Magn., vol. 42, pp. 3719-3729, doi: 10.1109/TMAG.2006.882308.

Baños A (1966), Dipole Radiation in the Presence of a Conducting Half Space. Oxford, U.K.: Pergamon.

Cella U M, Johnstone R, Shuley N (2009), "Electromagnetic wave wireless communication in shallow water coastal environment: Theoretical analysis and experimental results," in Proc. 4th ACM Int. Workshop UnderWater Netw., pp. 9:1-9:8, doi: $10.1145 / 1654130.1654139$

Conway J T (2007), "Inductance calculations for noncoaxial coils using Bessel functions," IEEE Trans. Magn., vol. 43, pp. 1023-1034, doi: 10.1109/TMAG.2006.888565.

Domingo M C (2012), "Magnetic induction for underwater wireless communication networks," IEEE Trans. Antennas Propag., vol. 60, pp. 2929-2939, doi: 10.1109/TAP.2012.2194670.

Hurley W G, Duffy M C (1995), "Calculation of self and mutual impedances in planar magnetic structures," IEEE Trans. Magn., vol. 31, pp. 2416-2422, doi: 10.1109/20.390151.

King R W P, Owens M, Wu T T (1992), Lateral Electromagnetic Waves. New York, NY, USA: Springer-Verlag.

Parise M (2015), "On the surface fields of a small circular loop antenna placed on plane stratified earth," Int. J. Antennas Propag., vol. 2015, 187806, doi: $10.1155 / 2015 / 187806$.

Piessens R, de Doncker-Kapenga E, Überhuber C W, Kahaner D K (1983), Quadpack: A Subroutine Package for Automatic Integration (Springer Series in Computational Mathematics). Berlin, Germany: Springer.

Piessens R (2000), "The Hankel transform," in Transforms and Applications Handbook (Electrical Engineering Handbook), Poularikas A Ed. Boca Raton, FL, USA: CRC Press, ch. 9.

Wait J R (1996), Electromagnetic Waves in Stratified Media (IEEE/OUP Series on Electromagnetic Wave Theory), Anderson J B, Ed. Piscataway, NJ, USA: IEEE Press.

Weaver R (2020), Numerical Methods for Inductance Calculation. Accessed: Mar. 6, 2020 [Online]. Available: http://electronbunker.ca/eb/CalcMethods3b.htm 